\title{
FLUCTUACIÓN DE PITYOPHTHORUS EICHHOFF (CURCULIONIDAE: SCOLYTINAE) CAPTURADOS EN TRAMPAS CEBADAS CON FEROMONAS EN EL BOSQUE LA PRIMAVERA, JALISCO
}

\author{
DYNAMIC POPULATION OF PITYOPHTHORUS EICHHOFF (CURCULIONIDAE: \\ SCOLYTINAE) CAUGHT BY TRAPS BAITED WITH PHEROMONES IN THE FOREST LA \\ PRIMAVERA, JALISCO
}

\author{
Sara Gabriela DÍAZ-RAMOS, ${ }^{1}$ Armando EQUIHUA MARTíneZ, ${ }^{1}$ Antonio RODRÍGUEZ RIVAS, ${ }^{2}$ Jorge \\ VALDEZ CARRASCO, ${ }^{1}$ OBdULIA LOURDES SEGURA-LEÓN ${ }^{1}$ y ThOMAS H. ATKINSON ${ }^{3}$ \\ ${ }^{1}$ Posgrado Fitosanidad, Colegio de Postgraduados, Campus Montecillo. Km 36.5 Carretera México-Texcoco, \\ 56230, Montecillo, Texcoco, Estado de México, México saragdiaz@hotmail.com, \\ equihuaa@colpos.mx,jvaldez@colpos.mx,sleon@colpos.mx. \\ 2 Departamento de Madera Celulosa y Papel arodrig@dmcyp.cucei.udg.mx. \\ ${ }^{3}$ Texas Natural History Collections, 3001 Lake Austin Blvd., Austin, TX 78703, U.S.A. \\ thatkinson.austin@gmail.com. \\ Recibido: 09/02/2016; aceptado: 06/06/2016 \\ Editor responsable: Alfonso N. García Aldrete
}

\begin{abstract}
Díaz-Ramos, S. G., Equihua. M. A., Rodríguez-Rivas, A., ValdezCarrasco, J., Segura-León, O. L., \& Atkinson, T. H. (2016). Fluctuación de Pityophthorus Eichhoff (Curculionidae: Scolytinae) capturados en trampas cebadas con feromonas en el bosque La Primavera, Jalisco. Acta Zoológica Mexicana (n.s.), 32(3), 296-299.
\end{abstract}

RESUMEN. Se realizó el monitoreo para Scolytinae con 10 trampas Lindgren de 8 embudos, cebadas con feromona frontalina + kairomona (alfa-pineno) del 2010 a 2012, cinco trampas en plantaciones de pino y cinco en bosque natural de encino-pino, en el Bosque La Primavera. Los insectos atraídos a las trampas fueron especies de Pityophthorus. Se identificaron siete especies, de las cuales Pityophthorus obtusipennis Blandford, P. occlusus Bright, y P. segnis subopacus Blackman, se reportan como nuevos registros para Jalisco, con densidades bajas. Las especies más abundantes fueron P. cacuminatus Blandford, con 719 individuos, con periodos de vuelo en época de poca precipitación, $P$. exquisitus (Blackman) especie no asociada a pinos, con 105 individuos con tendencia parecida a P. cacuminatus y P. schwerdtfegeri Schedl, especie mielófaga con 22 individuos, que se capturó en periodos con precipitaciones de 50 a $100 \mathrm{~mm}$.

Palabras clave: Scolytinae, precipitación, Pityophthorus cacuminatus.

\section{INTRODUCCIÓN}

Los descortezadores (Coleoptera: Curculionidae: Scolytinae) es uno de los grupos más diversos de Scolytinae, distribuidos en casi todo el mundo, asociados a una gran variedad de plantas (Raffa et al., 2015). Algunas especies
Díaz-Ramos, S. G., Equihua. M. A., Rodríguez-Rivas, A., ValdezCarrasco, J., Segura-León, O. L., \& Atkinson, T. H. (2016). Dynamic population of Pityophthorus Eichhoff (Curculionidae: Scolytinae) caught by traps baited with pheromones in the forest La Primavera, Jalisco. Acta Zoológica Mexicana (n.s.), 32(3), 296-299.

ABSTRACT. Monitoring of Scolytidae was carried out with 10 Lindgren traps of eight units baited with frontalin and kairomone + (alphapinene), from 2010-2012, with five traps in pine plantations and five traps in natural oak-pine forest at La Primavera forest. Insects attracted to the traps were Pityophthorus species. Seven species were identified, of which Pityophthorus obtusipennis Blandford, P. occlusus Bright and $P$. segnis subopacus Blackman, are new records for Jalisco, with low densities. The most abundant species were $P$. cacuminatus Blandford with 719 specimens, with flight periods in the low rainfall season, $P$. exquisitus (Blackman) not associated with pines, with 105 individuals, with similar trend to P. cacuminatus, and P. schwerdtfegeri Schedl myelophagous species with 22 individuals, captured in periods of rainfall from 50 to $10 \mathrm{~mm}$.

Key words: Scolytinae, rainfall. Pityophthorus cacuminatus

provocan serios daños en plantaciones agrícolas o forestales y bosques naturales; su distribución espacial dentro del hospedero va desde raíz hasta ramillas (Wilkinson \& Haack, 1987). De los insectos que afectan ramillas se encuentra el género Pityophthorus Eichhoff. La mayoría de las especies se consideran descortezadores verdaderos (se 
alimentan de floema), aunque algunas especies son mielófagas (barrenadores de la médula de ramillas) (Bright, 1981). Los ataques o barrenaciones al hospedero son iniciados por el macho, que atrae de tres a cinco hembras. Después del apareamiento, cada hembra forma una galería individual que se forma alrededor de la cámara central (Bright, 1981; Cibrián et al., 1995). Según Bright (1981) se pueden presentar varias generaciones en el mismo hospedero, pero no se conoce con exactitud. Pityophthorus tiene 385 especies a nivel mundial, con una mayor diversidad en las regiones Neártica y Neotropical (Hulcr et al., 2015). En México se registran 131 especies, de las que 26 están en Jalisco y de ellas diez se asocian con pino (Bright, 1981; Wood, 1982; Burgos-Solorio \& Equihua, 2007). Los daños ocasionados por Pityophthorus son aislados, porque arriban en ramillas de árboles débiles que fueron afectados por incendios, nevadas, sequías o ataques por descortezadores primarios (Cibrián et al., 1995), en California tres especies son vectores del cancro resinoso ( $F u$ sarium circinatum) (Erbilgin et al., 2005; Storer et al., 2004; Sakamoto et al., 2007).

El monitoreo de descortezadores mediante el uso de trampas cebadas con feromonas, es una forma de inferir la dinámica de la población, y con ello tomar medidas preventivas o de control. Para el género Dendroctonus, se han realizado estudios por el impacto económico que representa en bosques de coníferas, y en parte de ellos se han estudiado sus fluctuaciones poblacionales mediante trampas cebadas con feromonas de agregación (Rodríguez-Ortega et al., 2010; Vázquez et al., 2007). La frontalina es uno de los semioquímicos que se utilizan en Norte América para el monitoreo de Dendroctonus spp., y al que se le adiciona alfa-pineno para aumentar su fuerza de atracción, incluyendo descortezadores secundarios (Werner \& Holsten, 2002, Macías et al., 2004).

Este trabajo se enfocó inicialmente al monitoreo de Dendroctonus; el registro de este género fue nulo, pero otras especies de escolitinos fueron atraídos a las trampas. El género Pityophthorus fue el más común en el trampeo, por lo que el objetivo de este estudio es conocer la fluctuación poblacional de Pityophthorus spp., en el bosque La Primavera, Jalisco.

\section{MATERIALES Y MÉTODOS}

El estudio se realizó en el Campo Experimental Bosque Escuela (CEBE), en Cuxpala, Tala, Jalisco, en la parte suroeste del área protegida de flora y fauna La Primave- ra (20 34' $55.43^{\prime}$ 'N: $103^{\circ} 38^{\prime} 24.7^{\prime}$ 'O); cuenta con una superficie de 672 ha, presenta una distribución altitudinal entre 1350-1580 msnm, con un clima (A)Ca(wo)(w)e. Los tipos de vegetación incluyen bosque encino-pino, bosque tropical caducifolio, pastizal natural, vegetación acuática y subacuática. Además existen plantaciones de pino (Pinus oocarpa, $P$. douglasiana, $P$. devoniana y $P$. pseudostrobus) establecidas desde hace 30 años, con una superficie aproximada de 10 ha.

La red de monitoreo para descortezadores se estableció de enero de 2010 a diciembre de 2012. Se colocaron diez trampas tipo Lindgren de ocho embudos, cebadas con feromona de agregación frontalina Phero Tech Inc. más kairomona (alfa-pineno). El bote receptor contenía etanol al 70\%. Se ubicaron cinco trampas en plantaciones de pino y cinco en bosque natural de encino-pino. Las trampas se colocaron a una altura de $3 \mathrm{~m}$, sostenidas entre dos árboles, la distancia entre ellas fue de 300 a $400 \mathrm{~m}$. La recolección de los insectos se realizó quincenalmente, y los especímenes fueron colocados en etanol al 70\% para su conservación e identificación posterior. La feromona y la kairomona fueron reemplazadas mensualmente.

Para la determinación de los especímenes colectados se emplearon las claves especializadas de Bright (1981) y Wood (1982), y se analizaron características distintivas, tales como ornamentaciones del pronoto y dorso, presencia o ausencia de carina en la frente y el declive elitral.

Para la fluctuación de Pityophthorus se generó una gráfica de las especies más abundantes colectadas mensualmente en los tres años, con respecto a la precipitación

\section{RESULTADOS}

La colecta de Pityophthorus por tres años registró un total de siete especies con 856 individuos, seis de ellas asociados a pinos y una especie no asociada con pinos $(P$. exquisitus [Blackman] 1942). Del trampeo establecido en plantaciones de pino se encontraron seis especies con 440 individuos; para el bosque natural se encontraron cinco especies, con 425 individuos.

Para establecer la fluctuación de Pityophthorus en el bosque La Primavera, se consideraron tres especies con más capturas de individuos, tomándose en cuenta la precipitación, debido a que presentaba una mayor relación con la abundancia de los insectos (Fig. 1). La especie más abundante fue $P$. cacuminatus Blandford, con 719 individuos, seguida de $P$. exquisitus con 105 y $P$. schwerdtfege- 


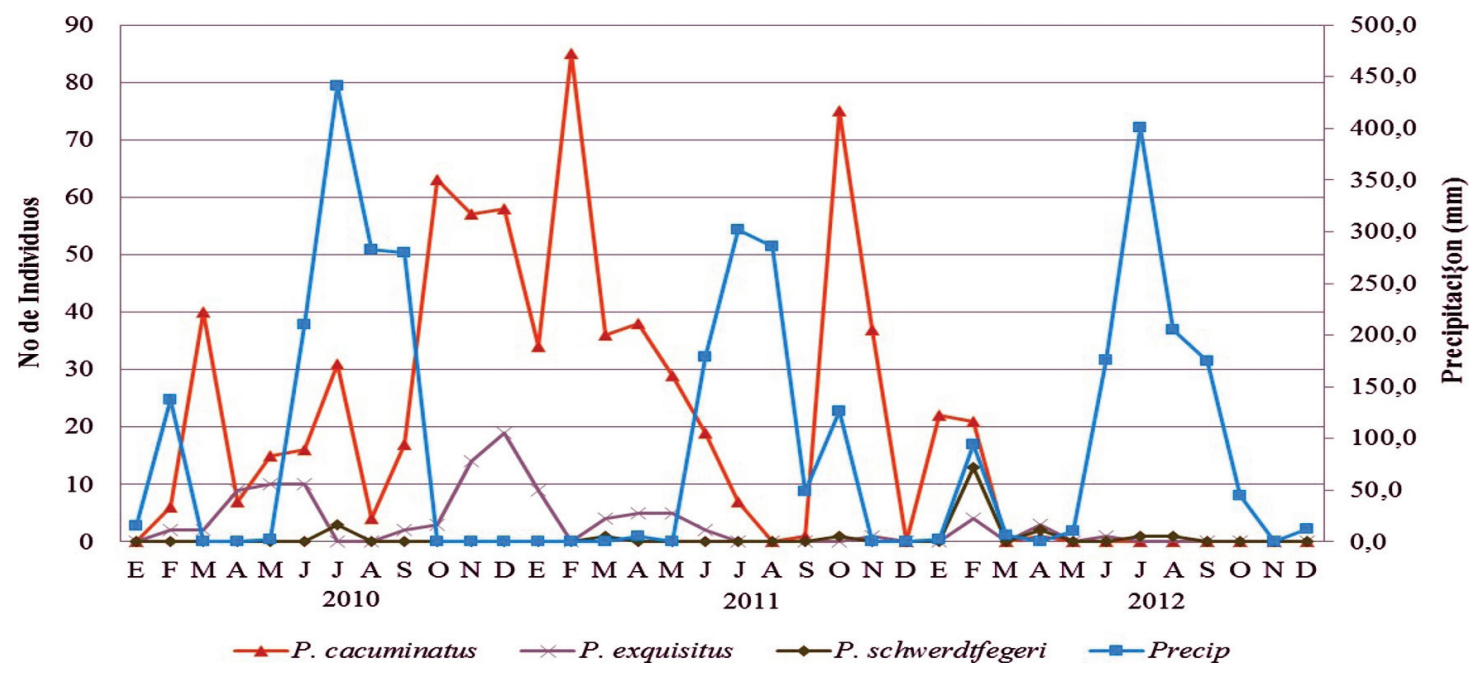

Figura 1. Fluctuación poblacional de las especies más abundantes de Pityophthorus, capturadas en el CEBE del Bosque La Primavera. Tala, Jalisco, 2010-2012.

ri Schedl, con 22 (Fig. 1). Las especies con bajas capturas fueron $P$. scabridus Schedl con un individuo, los nuevos registros para Jalisco son $P$. segnis subopacus Blackman, con 13 individuos, $P$. occlusus Bright, con 4 individuos y P. obtusipennis Blandford, con un individuo.

\section{DISCUSIÓN}

Para estos descortezadores se tiene detectada una feromona específica (Pityol), sin embargo fueron atraídos por la trampas cebadas con frontalina + alfa-pineno. Brauner \& Groot (2007) mencionan que Pityophthorus puberulus (LeConte) es atraído por alfa y beta-pineno, compuestos que se encuentran presentes en el floema de pinos. También el alfa-pineno es utilizada como sinergia con otras feromonas, lo que aumenta la atracción de los descortezadores entre ellos (Werner \& Holsten, 1984).

Pityophthorus cacuminatus fue la especie más abundante en los tres años de muestreo. Bright (1981) y Wood (1982) incluyen entre sus hospederos a Pinus oocarpa Schiede ex Schltdl; este pino es el dominante para el bosque La Primavera. En las dos coberturas vegetales del monitoreo $P$. cacuminatus fue la más colectada en plantación con 385 individuos y para bosque natural con 334; en el comportamiento de esta especie en los tres años de muestreo destacan seis picos, de los cuales en 2010 presentó tres, decreciendo en los años posteriores: 2011 (dos picos) y 2012 (1 pico) (Fig. 1). Lo que es evidente en la figura, es que la especie tiende a disminuir con la precipitación.
Para Pityophthorus exquisitus, especie no asociada a Pinus, se capturaron 77 individuos en bosque natural y solo 28 individuos en plantación. Esta especie presentó dos picos en primavera e invierno, con periodos de vuelo de tres cada uno en el año 2010; en 2011 apareció cuatro meses continuos en la estación de primavera lo que corresponde a un solo periodo de vuelo para ese año, en 2012 tiende a disminuir totalmente. Esta especie tiende a reducir sus densidades con la presencia de lluvias, la tendencia es parecida a $P$. cacuminatus, sin embargo con menos individuos capturados. Según Bright (1981) P. exquisitus está asociada al género Sambucus (Adoxaceae); Burgos y Equihua (2007) lo reportan en Fabaceae, Atkinson (2016) menciona que se ha colectado en especies de las familias Asteraceae y Betulaceae: en la zona de encino-pino se encuentran arbustos de la familia Asteraceae, en cuanto a las plantaciones los arbustos cercanos son escasos.

Pityophthorus schwerdtfegeri es una especie mielófaga, fue colectada únicamente en plantaciones en 2012 mostrando su densidad más alta en invierno; su presencia se asocia a una baja precipitación de 50-100 mm. Bright (1981) reporta a Pinus oocarpa como uno de sus hospederos.

Entre las especies menos abundantes, se capturaron 10 individuos de P. segnis subopacus en plantación, y tres individuos en bosque natural. Bright (1981) reporta entre sus hospederos a Pinus michoacana. De P. occlusus se colectaron dos individuos en bosque natural y en plantación, Bright (1981) y Wood (1982) reportan como hospedero a P. oocarpa y P. caribaea. Para P. scabridus se reporta un 
solo individuo en plantación, entre los hospederos se tiene a Pinus oocarpa, P. ayacahuite, P. montezumae, P. patula y P. pseudostrobus (Bright, 1981). La otra especie que presentó un solo individuo fue $P$. obtusipennis, las especies de pino que se reportan como hospederos por Bright (1981) y Wood (1982) son Pinus lawsoni, P. leiophylla y $P$. strobus var., chiapensis; no se encuentran en la zona de muestreo, aunque ellos mencionan que pueden tener otras especies de Pinus como hospederos.

\section{CONCLUSIONES}

En el monitoreo realizado en los tres años, Pityophthorus cacuminatus fue la especie más abundante, se registraron seis especies en plantaciones, cinco en bosque natural, uno de estos no asociado a pino. En cuanto a la feromona frontalina+ kairomona (alfa-pineno) presentó una mayor atracción en plantaciones. Del trampeo total se reportan tres nuevos registros para Jalisco. Para algunas especies encontradas en este trabajo sus periodos de vuelo están determinados por presencia o ausencia de precipitación.

\section{LITERATURA CITADA}

Atkinson, T. H. (2016). Barkbeetles.info. http:/www.barkbeetles.info/index.php. Consultado enero, 2016.

Brauner, A. M., \& Groot, P. (2007). Response of Pityophthorus puberulus (Coleoptera: Curculionidae: Scolytinae) to Pheromones and Terpenes. The Canadian Entomologist 139, 742-747.

Bright, D. E. (1981). Taxonomic Monograph of the Genus Pityophthorus Eichhoff in North and Central America (Coleoptera: Scolytidae). Memoirs of the Entomological Society of Canada, No $118,378 \mathrm{p}$.

Burgos-Solorio, A., \& Equihua, M. (2007). Platypodidae y Scolytidae (Coleoptera) de Jalisco, México. Dugesiana, 14, 59-82.

Cibrián, T. D., Méndez, M. J. T., Campos, B. R., Yates III, O., \& Flores, J. (1995). Insectos Forestales de México. Universidad Autónoma de Chapingo, Estado de México, México. 450 pp.

Erbilgin, N., Storer, A. J., \& Wood, D. L. (2005). Colonization of Cut Branches of Five Coniferous Host of the Pitch Canker Fungus by Pityophthorus spp. (Coleoptera: Scolytide) in Central, Coastal California. Entomological Society of Canada, 137, 337-359.
Hulcr, J., Atkinson, T. H., Cognato, A. I., Jordal, B. J., \& McKenna, D. D. (2015). Morphology, Taxonomy, and Phylogenetics of Bark Beetles, pp. 41-84. In: Vega, F. E.; Hofstetter, R. W. (Eds.). Bark Beetle Biology and Ecology of Native and Invasive Species. United States, Elsevier.

Macías, S. J. E., Niño, D. A., Cruz, L. J. A., \& Altúzar, M. R. (2004). Monitoreo de Descortezador y sus Depredadores Mediante el uso de Semioquímicos. El Colegio de la Frontera del Sur. Tapachula, Chiapas México. 28 pp.

Raffa, K. F., Grégoire, J. C., \& Lindgren B. S. (2015). Natural History and Ecology of Bark Beetle, pp 1-40. In: Vega, F. E.; Hofstetter, R. W. (Eds.). Bark Beetle Biology and Ecology of Native and Invasive Species. United States, Elsevier.

Rodríguez-Ortega, A., Equihua-Martínez, A., Cibrián-Tovar, J., \& Estrada-Venegas, E. G. (2010). Fluctuación de Dendroctonus adjunctus Blandford (Curculioniade: Scolytinae) y sus depredadores atraídos por Frontalina+Alfa-Pineno, la Estación Experimental de Zoquiapan, Edo. De México. Boletín del Museo de Entomología de la Universidad del Valle, 11, 20-17.

Ruiz, C. J. A., Flores, L. H. E., Regalado, R. J. R., \& Ramírez, O. G. (2012). Estadísticas Climáticas Normales del Estado de Jalisco. Libro Técnico. Núm. 2, INIFAP-CIRPAC- Campo Experimental Centro Altos de Jalisco. Tepatitlán de Morelos. Jalisco. $350 \mathrm{pp}$.

Sakamoto, J. M., Gordon, T. R., Storer, A. J., \& Wood, D. L. (2007). The Role of Pityophthorus spp. As vector of pitch canker affecting Pinus radiata. The Canadian Entomologist, 139, 864-871.

Storer, A., Wood, D. L., \& Gordon T. R. (2004). Twig beetles, Pityophthorus spp. (Coleoptera: Scolitydae), as vector of the pitch canker pathogen in California. The Canadian Entomologist, 136, 685-693.

Vázquez, C. I., Sánchez-Martínez, G., \& Madrigal, H. S. (2007). Fluctuación Poblacional de Dendroctonus mexicanus Hopk., bajo dos Condiciones de Manejo Forestal en Michoacán, México. Revista Ciencia Forestal en México, 32, 55-77.

Werner, E. A., \& Holsten E. H. (1984). Scolytidae Associated with White Spruce in Alaska. Canadian Entomologist, 116, 465-471.

Werner, R.A., \& Holsten, E. H. (2002). Use of semiochemicals of secondary bark beetles to disrupt spruce beetle attraction and survival in Alaska. Research Paper PNW-RP541. Portland, OR. United States Departament of Agriculture, Forest Service, Pacific Northwest Research Sation. 11 p.

Wilkinson, R. C., \& Haak R. A. (1987). Within-tree distribution of pine bark beetle (Coleoptera: Scolytidae) in Honduras. Ceiba, 28, 155-133.

Wood, S. L. (1982). The Bark and Ambrosia Beetles of North and Central America (Coleoptera: Scolytidae), a Taxonomic Monograph. Great Basin Naturalist Memoirs, 6, 1359 pp. 